\title{
Induced production of anti-etanercept antibody in collagen-induced arthritis
}

\author{
HYOJU YI, JURYUN KIM, HYERIN JUNG, YERI ALICE RIM, YOUNGKYUN KIM, \\ SEUNG MIN JUNG, SUNG-HWAN PARK and JI HYEON JU
}

\begin{abstract}
Division of Rheumatology, Department of Internal Medicine, CiSTEM Laboratory, Convergent Research Consortium for Immunological Disease (CRCID), Seoul St. Mary's Hospital, College of Medicine, The Catholic University of Korea, Seoul 137-701, Republic of Korea
\end{abstract}

Received September 17, 2013; Accepted March 12, 2014

DOI: $10.3892 / \mathrm{mmr} .2014 .2127$

\begin{abstract}
Etanercept is a widespread biological drug for the treatment of rheumatoid arthritis, which inhibits tumor necrosis factor- $\alpha$ (TNF- $\alpha)$. Recently, the presence of antibodies targeting TNF- $\alpha$ inhibitors such as infliximab and adalimumab, was reported. However, few reports have studied etanercept in a mouse model of arthritis. We investigated the induction of anti-etanercept antibody production, along with the antibody's potential interfering effects on the biological function of etanercept, in mice with collagen-induced arthritis (CIA). CIA mice received an intraperitoneal injection of etanercept (25, 100 or $400 \mu \mathrm{g}$ per mouse). The degree of inflammation and cartilage erosion was evaluated, and the number of osteoclasts in the ankle joints was assessed by TRAP staining. The level of pro-inflammatory cytokines in the serum was measured. To analyze the anti-osteoporotic effect of etanercept, microfocal computed tomography analyses of femurs and tibias were performed. Etanercept treatment decreased both the incidence and severity of arthritis in a dose-dependent manner, except for the highest dose of $400 \mu \mathrm{g}$. The mice that were treated with 25 and $100 \mu \mathrm{g}$ etanercept showed an improvement in inflammation, cartilage damage, and even bone loss. However, mice treated with $400 \mu \mathrm{g}$ etanercept showed no significant improvement in any of the tested parameters. Using a customized enzyme-linked immunosorbent assay (ELISA), the presence of the anti-etanercept antibody was detected in the serum in this treatment-refractory group. The therapeutic effect of etanercept was reduced in the CIA mice that developed the anti-etanercept antibody. In conclusion, the production of an anti-etanercept antibody can be induced in CIA mice, and this antibody can considerably reduce the anti-arthritic and anti-osteoporotic effects of etanercept.
\end{abstract}

Correspondence to: Professor $\mathrm{Ji}$ Hyeon Ju, Division of Rheumatology, Department of Internal Medicine, Seoul St. Mary's Hospital, The Catholic University of Korea, 505 Banpodong, Seochogu, Seoul 137-701, Republic of Korea

E-mail: juji@catholic.ac.kr

Key words: etanercept, anti-drug antibody, collagen-induced arthritis, rheumatoid arthritis, tumor necrosis factor- $\alpha$

\section{Introduction}

Rheumatoid arthritis (RA) is a chronic inflammatory autoimmune disease, associated with focal and systemic bone loss. Structural changes such as joint damage and osteoporosis are the most suitable symptoms for distinguishing RA from other diseases (1). The level of tumor necrosis factor- $\alpha$ (TNF- $\alpha$ ) is markedly increased in most patients with RA, and thus, the protein is considered a main pathological player in inflammation. As a result, many TNF- $\alpha$ inhibitors, including infliximab (Remicade ${ }^{\circledR}$ ), adalimumab (Humira ${ }^{\circledR}$ ), and etanercept $\left(\right.$ Enbrel $\left.^{\circledR}\right)$, were developed and have been widely used to treat RA $(2,3)$.

Etanercept, one of the most commonly used TNF- $\alpha$ inhibitors, consists of two human TNF receptor 2 (TNFR2) extracellular domains conjugated to the Fc portion of the human IgG1. The anti-inflammatory effect of etanercept in patients with RA is widely accepted (4-7). Although a previous study indicated that inhibiting inflammation in RA is insufficient to inhibit bone destruction (8), another study showed that TNF- $\alpha$ blockers directly mitigate osteoporosis (9). It was also suggested that TNF- $\alpha$ inhibitors prevent resorption of the bone adjacent to the joints (10).

Despite the efficiency of TNF- $\alpha$ inhibition in RA treatment, it is commonly not feasible to prescribe TNF- $\alpha$ inhibitors to patients with early RA, due to its high cost and insurance regulations $(3,11)$. British guidelines require limitation of prescriptions of TNF- $\alpha$ inhibitors to patients with active RA who do not have a satisfactory response to at least two disease-modifying antirheumatic drugs (DMARDs) (12). However, since it has been reported that etanercept is effective when administered to patients with early RA $(5,13)$, the importance of using this drug in the treatment of early RA is being reassessed (14). Furthermore, there has been limited clinical research on the anti-osteoporotic effects of TNF- $\alpha$ inhibitors in patients with early RA. In this study, we simulated this clinical condition in mice by transiently injecting etanercept at the early stage of CIA induction.

Since TNF inhibitors have become popular drugs for the treatment of RA, clinicians are increasingly interested in the subgroup of the patients who show a refractory response to etanercept. One explanation for refractoriness is the 
development of anti-drug antibody (ADA) against the protein, as part of the body's physiological reaction. Not only the mouse chimeric form of the monoclonal antibody, infliximab, but also humanized drugs such as adalimumab, are reported to induce the development of ADA. However, etanercept is less known to induce ADA and less studied in comparison to infliximab and adalimumab.

In this study, we established a mouse arthritic model in which ADA production was investigated at various doses of etanercept challenge. Furthermore, we investigated whether mice with ADA show reduced focal and systemic osteoporosis as well as inflammation, and explored the underlying mechanisms. In addition, we studied the effect of etanercept in early RA by injecting CIA mice with etanercept during the early stages of RA.

\section{Materials and methods}

Induction of CIA and assessment of severity. All procedures on animals were in accordance with the Laboratory Animals Welfare Act, the Guide for the Care and Use of Laboratory Animals, and the Guidelines and Policies for Rodent Experiments provided by the Institutional Animal Care and Use Committee (IACUC) in the School of Medicine, The Catholic University of Korea [Catholic University Medical College (CUMC-2011-0010-03)]. Male DBA1/J mice (6 weeks old; Orient Bio Inc, Seongnam, Korea) were immunized by intradermal injection into the base of the tail of bovine type II collagen (100 $\mu \mathrm{g} / \mathrm{mouse}$; Chondrex, Inc., Redmond, WA, USA), emulsified in Freund's complete adjuvant (Arthrogen-CIA ${ }^{\circledR}$, Chondrex, Inc). CIA symptoms were evident between 17 and 21 days following the first immunization. The incidence and severity of CIA were monitored and scored as previously described (15). The CIA incidence was expressed as the percentage of swollen paw in the 4 paws of each mouse.

Etanercept treatment. Mice with CIA were given an intraperitoneal injection of either phosphate-buffered saline (PBS) or 25, 100 and $400 \mu$ g etanercept (Enbrel ${ }^{\circledR}$; Pfizer, New York, NY, USA) 3 times/week, from day 7 to day 28 after the first immunization. No additional treatment was provided until sacrifice, performed at week 10 after the first immunization.

Histological evaluation of arthritis. The hind leg of each mouse was isolated and fixed in $10 \%$ formalin. After decalcification in hydrochloric acid, samples were embedded in paraffin. The sections were stained with hematoxylin and eosin (H\&E), safranin $\mathrm{O}$, and toluidine blue. The degree of inflammation was determined based on H\&E staining using the following scoring scheme: 0 , no inflammation; 1 , mild thickening of the lining layer or some infiltrating cells in the sublining layer; 2, mild thickening of the lining layer and some infiltrating cells in the sublining layer; 3 , thickening of the lining layer, influx of cells in the sublining layer, and existence of cells in the synovial space; and 4, synovium highly infiltrated with numerous inflammatory cells. Cartilage damage was scored based on safranin $\mathrm{O}$ and toluidine blue staining according to the following scheme: 0 , no destruction; 1 , minimal erosion, limited to single spots; 2 , slight to moderate erosion in a limited area; 3 , more extensive erosion; and 4, general destruction. Tartrate-resistant acid phosphatase (TRAP) staining was performed using a commercial kit (Sigma-Aldrich, St. Louis, MO, USA) as described by the manufacturer, except for hematoxylin counterstaining. $\mathrm{TRAP}^{+}$multinucleated cells with $\geq 3$ nuclei were counted as osteoclasts. All histological assessments were performed by 2 independent blinded observers.

Quantification of cytokine levels. At 2 and 3 weeks following the first collagen immunization, $\sim 100 \mu \mathrm{l}$ of venous blood were taken from the orbital sinus of anesthetized mice. Following incubation at room temperature for $1 \mathrm{~h}$, blood samples were centrifuged for $20 \mathrm{~min}$ at $15,000 \mathrm{~g}$. Serum was transferred into new tubes and stored at $-80^{\circ} \mathrm{C}$. The sera were analyzed with a Milliplex ${ }^{\circledR}$ MAP Mouse Cytokine/Chemokine kit that uses a Luminex xMAP detection system (Millipore, Bedford, MA, USA). Quantification of data was performed using the Masterplex QT version 4.0 software (MiraiBio Inc., Tokyo, Japan).

Microfocal computed tomography (micro-CT) analyses. Micro-CT analyses of the distal femoral and proximal tibial metaphyses were performed with a desktop microcomputer tomography scanner (SkyScan 1172; Bruker-microCT, Kontich, Belgium). The samples were fixed in $3.7 \%$ formaldehyde for $>24 \mathrm{~h}$ and were scanned through a $0.5-\mathrm{mm}$-thick filter using a $141 \mu \mathrm{A}$ current, $70 \mathrm{kV}$ source voltage, and an exposure time of $590 \mathrm{msec}$. To consistently set the trabecular bone region range, data from each sample were resampled with the CTAn application following reconstruction of the scanned images with the NRecon application (both from BRUKER-MICROCT). The morphometric parameters percentage of bone volume, trabecular thickness, trabecular number, trabecular separation and bone surface density were measured by the CTAn application. Bone mineral density was measured in 77 continuous slices.

Measurement of serum anti-etanercept antibody levels. Sera were obtained from all mice at sacrifice, and the concentration of the anti-etanercept antibody was measured by enzyme-linked immunosorbent assay (ELISA) as previously described (16), with minor modifications. Briefly, $250 \mathrm{ng} / 50 \mu \mathrm{l}$ etanercept (0.1 M sodium carbonate, $\mathrm{pH} 9.6$ ) were coated on the surface of a microtiter plate overnight at $37^{\circ} \mathrm{C}$. The coated wells were blocked with $2 \%$ skim milk in PBS for $1 \mathrm{~h}$ at $37^{\circ} \mathrm{C}$ and rinsed with washing buffer (0.1\% Tween-20 in PBS). Serially diluted serum samples $(1: 1,000-1: 100,000)$ were added to the wells for $2 \mathrm{~h}$. The wells were then washed 3 times with washing buffer. Horseradish peroxidase (HRP)-conjugated anti-mouse IgG at a 1:5,000 dilution in PBS was added and incubated for $1 \mathrm{~h}$. After 5 washes with washing buffer, the tetramethyl benzidine reaction was initiated, and $\mathrm{H}_{2} \mathrm{SO}_{4}$ was used as the stop solution. The absorbance (optical density; OD) was measured at $450 \mathrm{~nm}$.

\section{Results}

Etanercept attenuates arthritis in CIA mice. Since bone damage starts at the early stage of the disease, it is advised to inject etanercept in CIA mice at the early stages to examine its anti-osteoporotic effect. Thus, we performed 9 
A

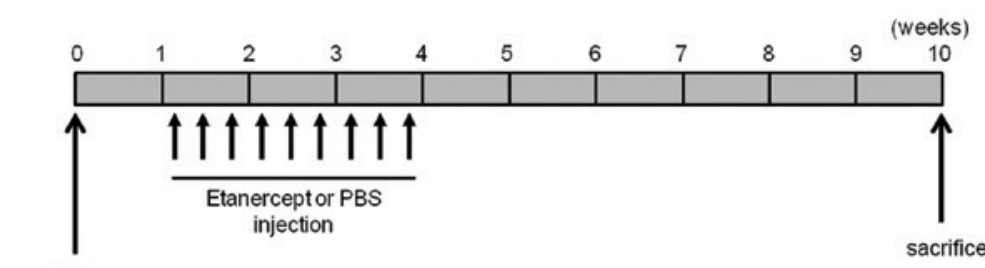

$$
\text { Cll } 1^{\text {st }}
$$

immunization

B

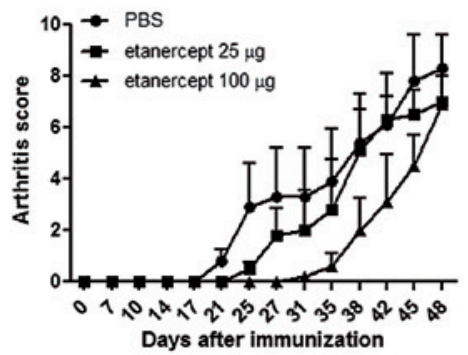

C

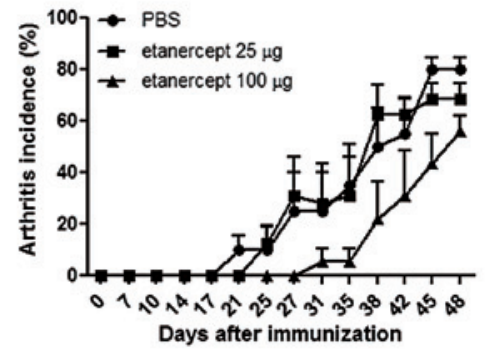

D

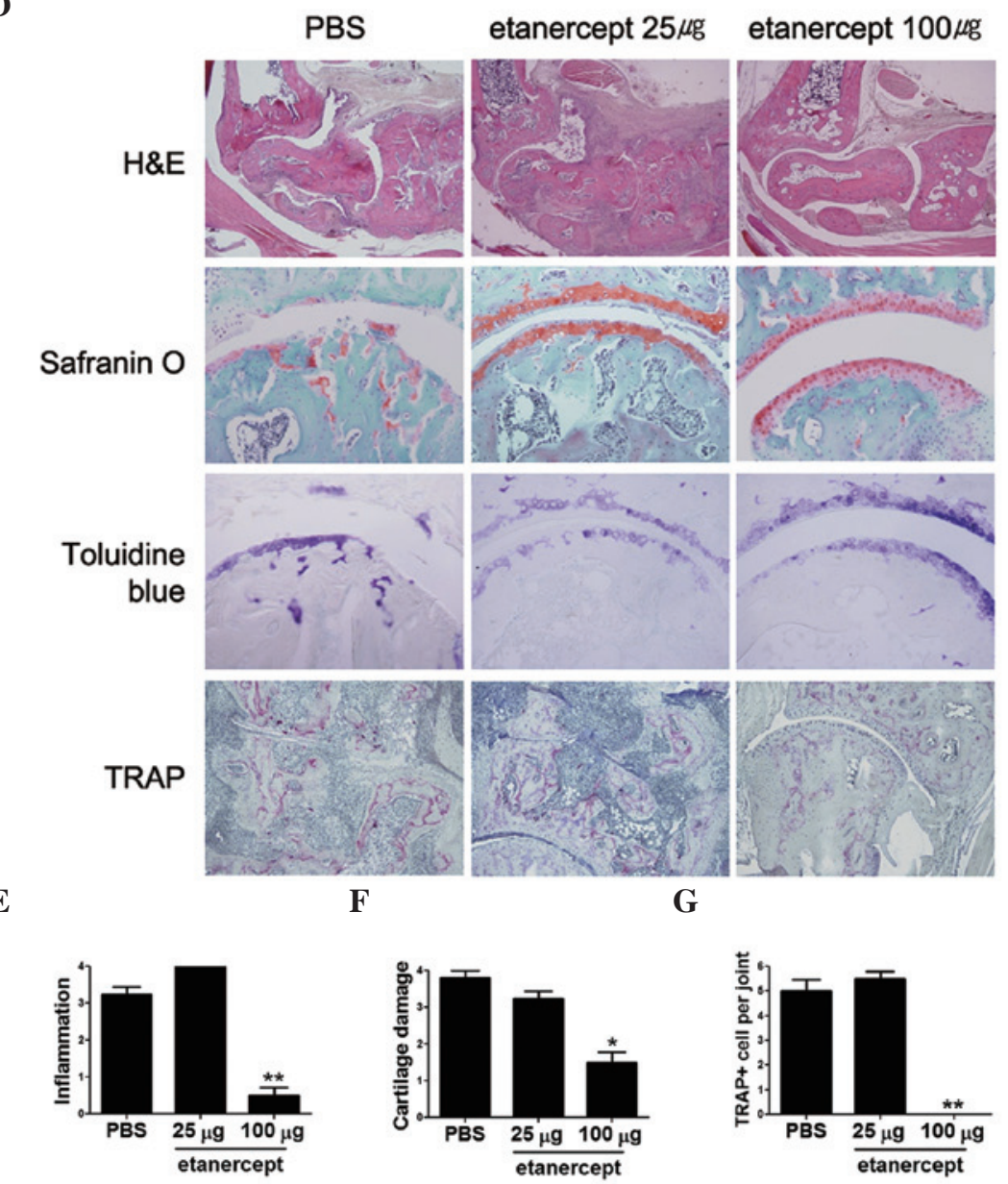

Figure 1. Etanercept alleviates arthritis in mice with collagen-induced arthritis (CIA). (A) The timeline for immunization and sacrifice of mice. (B) The inflammation of each paw was scored in a scale from 0 to 4 (with 4 being the most severe inflammation). The sum of scores for all 4 paws was used to calculate the arthritis score. (C) The incidence of arthritis was expressed as a percentage of swollen paw in the 4 paws of each mouse. Values are expressed as mean \pm SEM. (D) Ankle joint of a hind limb stained with hematoxylin and eosin (H\&E), safranin O, toluidine blue, and tartrate-resistant acid phosphatase (TRAP). (E) The extent of joint inflammation was determined after H\&E staining using the criteria described in Materials and methods. (F) Cartilage damage was scored based on safranin $\mathrm{O}$ and toluidine blue staining as described in Materials and methods. (G) TRAP ${ }^{+}$multinucleated cells with $\geq 3$ nuclei were counted. Values are expressed as mean \pm SEM. ${ }^{*} \mathrm{P}<0.05 ;{ }^{* * *} \mathrm{P}<0.01$ compared to the PBS-treated group. CII, bovine type II collagen.

intraperitoneal injections of etanercept to CIA mice, 3 times per week for 3 weeks, starting from day 7 after the first immunization (Fig. 1A), although the extent of inflammation commonly reaches a peak at week 8 in this model of arthritis. Throughout this period, we monitored the severity of arthritis in each group to examine whether etanercept injection 
A

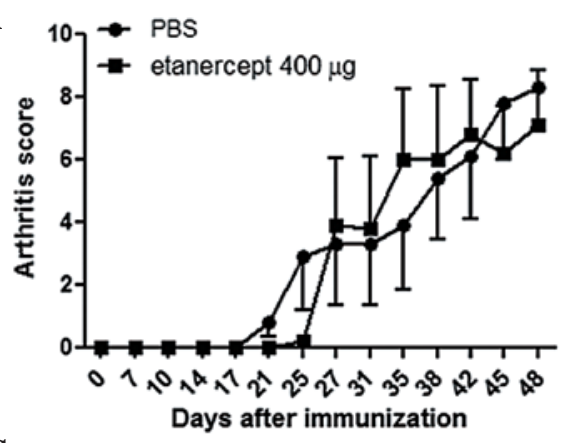

C

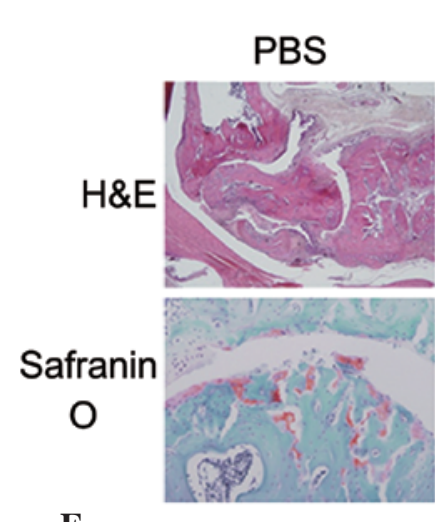

$\mathbf{E}$

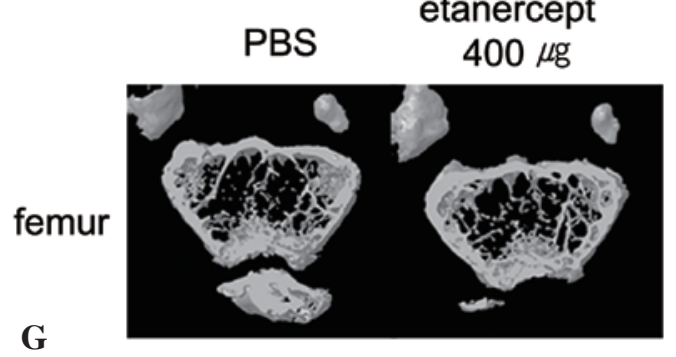

B

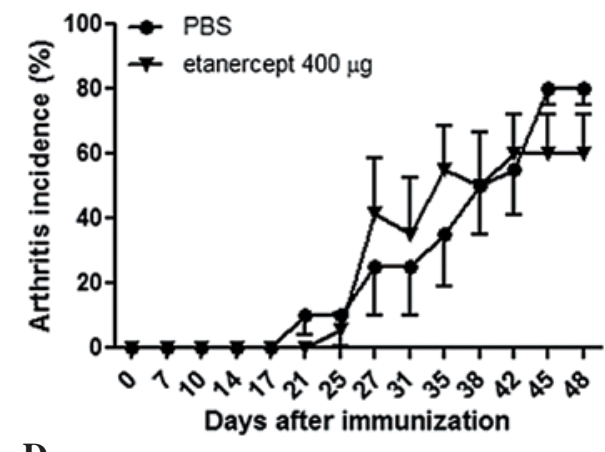

D

Days after immunization

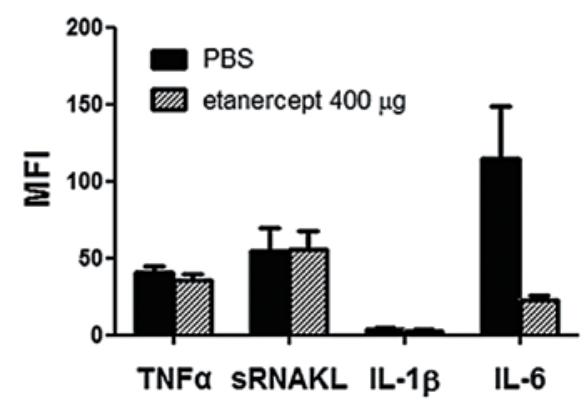

$\mathbf{F}$

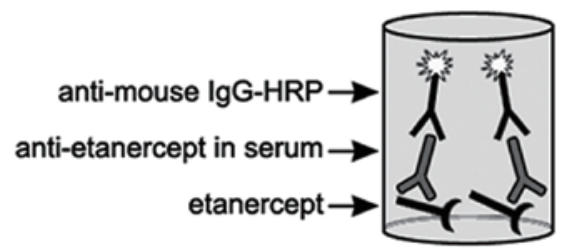

H

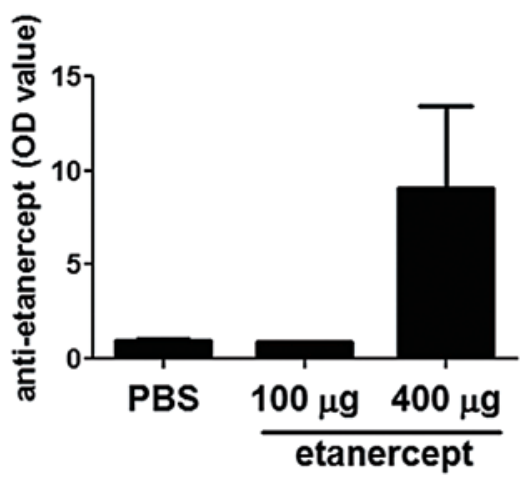

Figure 2. Production of the anti-etanercept antibody is induced in sera of mice treated with $400 \mu \mathrm{g}$ of etanercept, and which did not respond to this dose. (A) The inflammation of each paw was scored in a scale from 0 to 4 (with 4 being the most severe inflammation). The sum of scores for all 4 paws was used to calculate the arthritis score. (B) The incidence of arthritis was expressed as a percentage of swollen paw in the 4 paws of each mouse. Values are expressed as mean \pm SEM. (C) Ankle joint of a hind limb stained with hematoxylin and eosin (H\&E) and safranin O. (D) The levels of tumor necrosis factor- $\alpha$ (TNF- $\alpha$ ), soluble receptor activator of nuclear factor $\kappa-B$ ligand (sRANKL), interleukin (IL)-1 $\beta$, and IL-6 at week 2 after the first collagen immunization, expressed in mean fluorescence intensity (MFI) values. (E) Representative microfocal computed tomography image of femur from mice with collagen-induced arthritis (CIA) or mice treated with $400 \mu \mathrm{g}$ etanercept. (F) Schematic graph of the customized enzyme-linked immunosorbent assay (ELISA) used to measure the production of the anti-etanercept antibody in the serum using horseradish peroxidase (HRP)-conjugated IgG. (G and H) The amount of anti-etanercept antibody in the serum expressed as mean optical density (OD) \pm SEM.

mitigates inflammation during the early stages of CIA, and whether the degree of inflammation correlates with that of bone loss. The results demonstrated that mice that were injected with $100 \mu \mathrm{g}$ etanercept show a significant reduction in arthritic symptoms compared to those injected with
PBS (Fig. 1B and C), although the effects appeared to last for only 18 days after the injections.

Demineralized bone was stained with H\&E, safranin O, and toluidine blue to determine whether etanercept-mitigated inflammation resulted in a reduction of cartilage erosion in the 
A

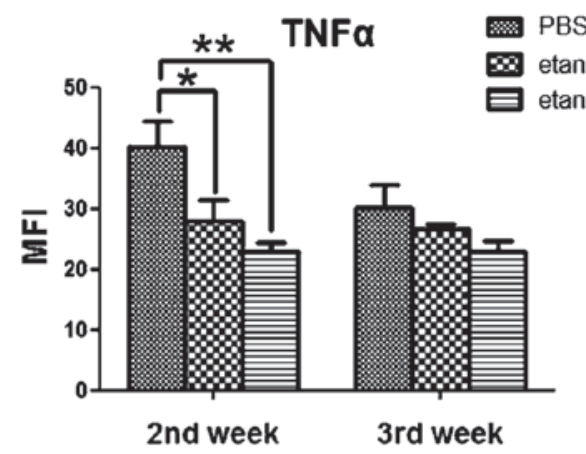

C

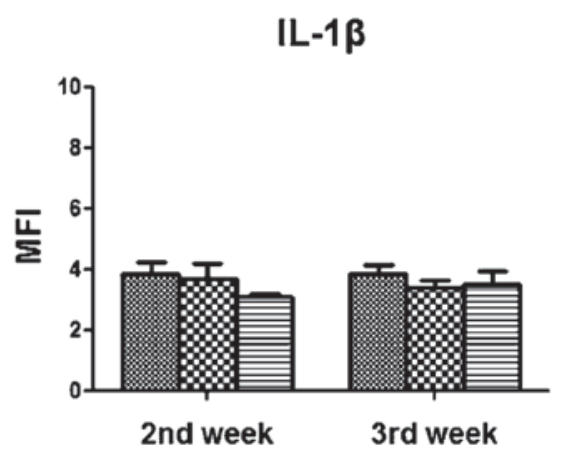

B

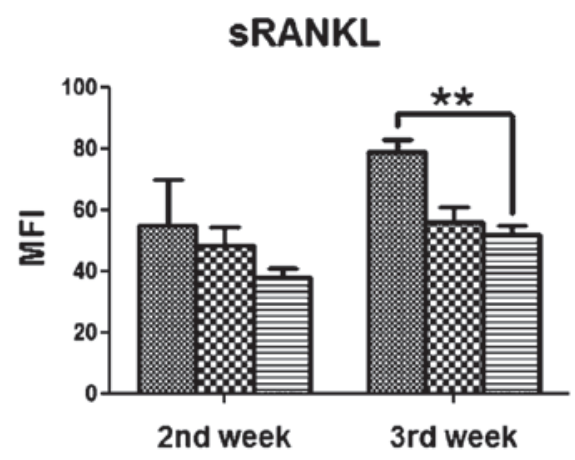

D

\section{IL-6}

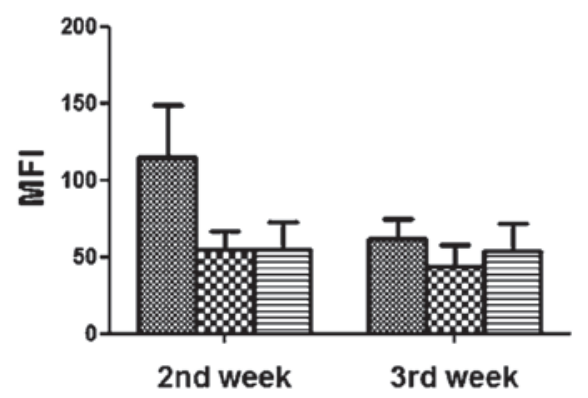

Figure 3. Levels of pro-inflammatory cytokines are affected by etanercept treatment. Mean fluorescent intensity (MFI) of (A) tumor necrosis factor- $\alpha$ (TNF- $\alpha$ ), (B) soluble receptor activator of nuclear factor $\kappa-B$ ligand (sRANKL), (C) interleukin (IL)-1 $\beta$ and (D) IL-6 at weeks 2 and week 3 following the first collagen immunization. Values are expressed as mean \pm SEM. ${ }^{*} \mathrm{P}<0.05 ;{ }^{* *} \mathrm{P}<0.01$ compared to the PBS-treated group.

metacarpophalangeal joints of the animals (Fig. 1D). The results showed that mice treated with $100 \mu \mathrm{g}$ etanercept display reduced infiltration and cartilage erosion compared to control CIA mice, consistent with the RA scores and incidence rates. Joint inflammation in the group treated with $100 \mu$ g etanercept was $\sim 1 / 7$ th of that observed in control CIA mice, and cartilage damage was as low as $35 \%$ of that observed in CIA mice (Fig. 1E and F). Although the effects were not as significant as those seen in mice treated with $100 \mu \mathrm{g}$ etanercept, the group treated with $25 \mu \mathrm{g}$ etanercept also showed a reduction in arthritis. In this case, the degree of inflammation was slightly higher compared to control CIA mice, but cartilage damage was reduced, indicating that $25 \mu \mathrm{g}$ of etanercept can prevent cartilage damage.

Since TNF- $\alpha$ is one of the key inducers of osteoclastogenesis (17), we assumed that etanercept treatment can reduce the number of osteoclasts, thus reducing bone damage. The bones were stained with TRAP to determine the number of multinucleated osteoclasts (Fig. 1G). No osteoclast was detected in the bones or joints of mice treated with $100 \mu \mathrm{g}$ etanercept, whereas a considerable number of osteoclasts were detected in joints of control CIA mice, and of mice treated with $25 \mu \mathrm{g}$ etanercept, consistent with the degree of cartilage damage in these groups.

Anti-etanercept antibody production is induced in mice challenged with high doses of etanercept. We performed the same experiments with a higher dose of etanercept, $400 \mu \mathrm{g}$. The mice treated with this dose showed little or no difference to PBS-treated mice (Fig. 2A-E). The levels of TNF- $\alpha$ and soluble receptor activator of nuclear factor $\kappa$-B ligand (sRANKL) in the serum of high-dose etanercept-treated mice were similar to those measured in the serum of control CIA mice (Fig. 2D).
Bone destruction and demineralization were prominent in this group (Fig. 2E). We measured the concentration of the anti-etanercept antibody at the time of sacrifice using ELISA (Fig. 2F). Notably, the anti-etanercept antibody was detected in mice treated with $400 \mu \mathrm{g}$ etanercept, even when serum samples were diluted by $1 / 1,000$ (Fig. 2G and $\mathrm{H}$ ).

Etanercept affects the level of pro-inflammatory cytokines in the serum of CIA mice. Since TRAP staining data showed that etanercept affects the number of osteoclasts in the ankle joints, we assumed that there may be differences in the levels of cytokines related to osteoclastogenesis in the sera of these mice. To examine whether etanercept treatment up- or downregulates TNF- $\alpha$, sRANKL, interleukin (IL)- $1 \beta$ and IL-6, we collected sera from the orbital sinus of anesthetized animals at the second and third week after the first collagen immunization. At 2 weeks after immunization, the serum levels of TNF- $\alpha$ and sRANKL were consistent with the results described in Fig. 1 (Fig. 3A and B). The lowest concentrations of TNF- $\alpha$ and sRANKL were found in the sera of mice treated with $100 \mu \mathrm{g}$ of etanercept. The dosages of 25, 100 and $400 \mu \mathrm{g}$ were compared. Among them $100 \mu \mathrm{g}$ was more effective than $25 \mu \mathrm{g}$ (Fig. 1), however, at $400 \mu \mathrm{g}$ showed no improvement (Fig. 2). TNF- $\alpha$ and sRANKL levels in the serum of mice treated with $25 \mu \mathrm{g}$ etanercept were lower compared to those measured in the serum of control CIA mice, but were higher compared to those measured in the serum of mice that received the most effective dose $(100 \mu \mathrm{g})$, indicating dose dependency. The IL-6 levels showed time-dependent variation; at the second week following the first collagen immunization, the highest IL-6 levels were observed in the serum of control CIA mice, but these levels were almost 
A

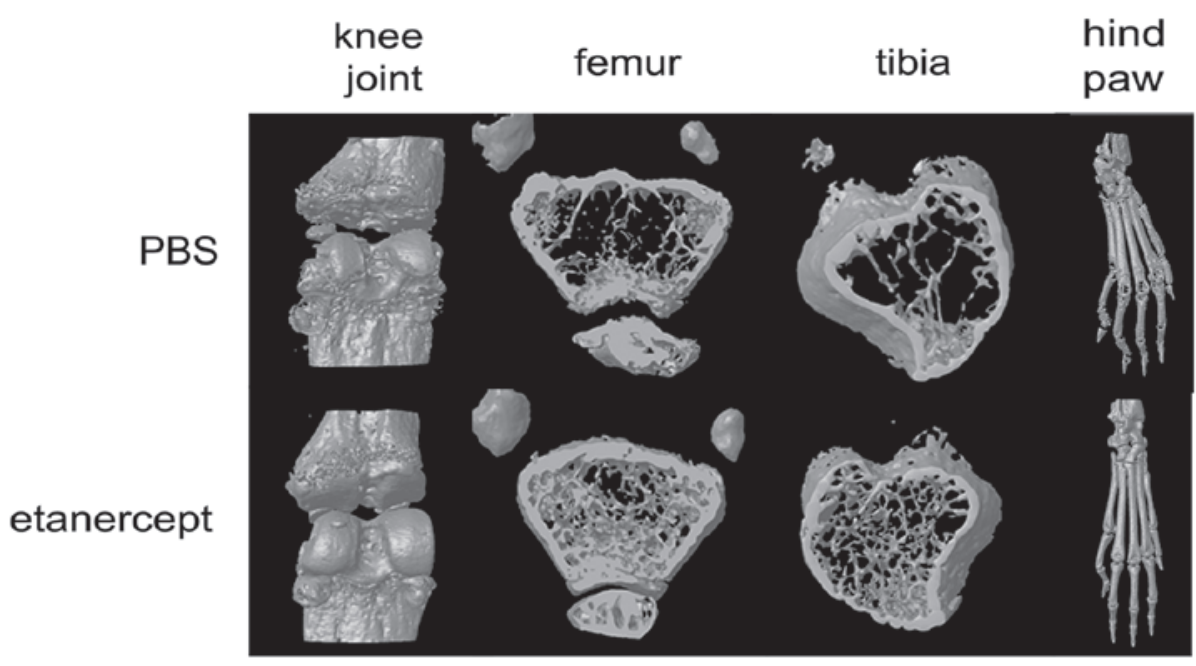

B
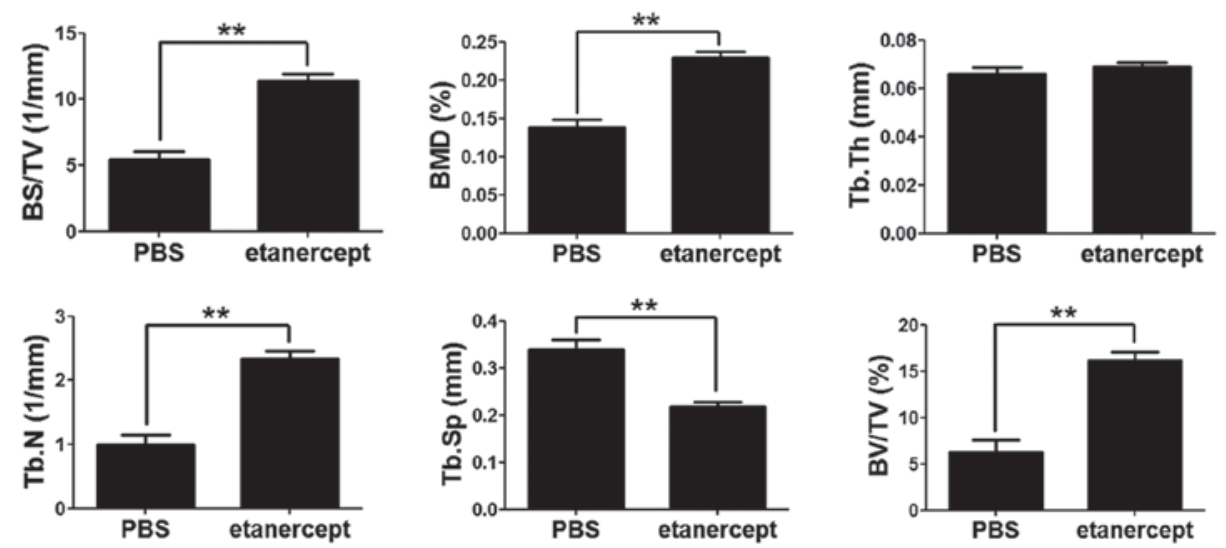

Figure 4. Etanercept has anti-osteoporotic effects in mice with collagen-induced arthritis (CIA). (A) Representative images of microfocal computed tomography (micro-CT) analyses of distal femoral and proximal tibial metaphyses. Knee joint, femur, tibia and hind paws from CIA and $100 \mu \mathrm{g}$ etanercept-treated mice are shown. (B) Changes in the morphometric parameters bone surface density (BS/TV), bone mineral density (BMD), trabecular thickness (Tb.Th), trabecular number (Tb.N), trabecular separation (Tb.Sp), and bone volume (BV/TV). Values are expressed as mean \pm SEM. ${ }^{* *} \mathrm{P}<0.01$ compared to the PBS-treated group.

identical in all groups by the third week. By contrast, the levels of IL-1 $\beta$ were relatively low throughout the experiment and did not significantly differ between groups (Fig. 3C and D). These data showed that the TNF- $\alpha$ and sRANKL levels in the sera are largely affected by etanercept treatment, and indicate that systemic bone loss may be regulated by etanercept, through inhibition of osteoclastogenesis.

Etanercept treatment at the early stage of disease prevents systemic bone demineralization. As our data showed, etanercept reduced osteoclastogenesis and affected the level of osteoclastogenesis-associated cytokines, TNF- $\alpha$ and sRANKL. We next investigated, using micro-CT analysis, whether etanercept can prevent systemic bone loss. The distal femoral and proximal tibial metaphyses from each mouse were amputated and analyzed by micro-CT to identify the effects of etanercept on bone demineralization, induced by arthritis (Fig. 4A and B). As expected, mice treated with a low dose of etanercept $(25 \mu \mathrm{g})$ did not show any considerable improvement of disease symptoms, and we therefore focused on mice treated with $100 \mu \mathrm{g}$ etanercept. Compared to the control CIA mice, the $100 \mu \mathrm{g}$ etanercept-treated group showed a 3-fold increase in the percentage of bone volume (BV/TV), $\sim 65 \%$ higher bone mineral density (BMD), nearly 2-fold higher bone surface density (BS/TV), $30 \%$ lower trabecular separation (Tb.Sp), and a 2-fold higher trabecular number (Tb.N). These results indicate that etanercept inhibits bone demineralization in this group (Fig. 4A and B). The three-dimensional images of knee joints and hind paws clearly illustrated this tendency (Fig. 4A). Trabecular thickness (Tb.Th) was similar in all groups. These data indicate that etanercept may attenuate systemic demineralization and focal erosion in CIA mice, even when it is administered at the early stage of the disease.

\section{Discussion}

In this study, we showed that etanercept can ameliorate both osteoporosis and inflammation in CIA mice. By treating with etanercept only during the early stage of arthritis, we measured how long the effect of etanercept can last and how important anti-TNF- $\alpha$ treatment is, in an experimental RA model (Fig. 1A). The anti-inflammatory effect of $100 \mu \mathrm{g}$ etanercept lasted $\sim 18$ days following cessation of treat- 
ment (Fig. 1B and C). The formation of pannus with bone erosion tended to correlate with the severity of swelling, showing that $100 \mu \mathrm{g}$ etanercept is effective against both inflammation and focal bone erosion in RA (Fig. 1D-G).

We examined differences in the sera cytokine levels of mice following etanercept treatment (Fig. 3). The results showed that SRANKL and TNF- $\alpha$ decreased as the disease ameliorated, suggesting that the effect of etanercept correlates with changes in these cytokines. Both SRANKL and TNF- $\alpha$ are well-known mediators of osteoclastogenesis; sRANKL combined with M-CSF is sufficient to induce osteoclastogenesis and has been widely used in in vitro osteoclast formation experiments (18-20). Moreover, TNF- $\alpha$ stimulates osteoclast differentiation independently of RANK-RANKL signaling (17). Therefore, the reduction of sRANKL and TNF- $\alpha$ levels in mice treated with $100 \mu \mathrm{g}$ etanercept may inhibit osteoclastogenesis, preventing the CIA-induced bone loss.

BMD was measured by micro-CT to investigate whether the effect of etanercept is consistent with a systemic reduction in bone erosion. Systemic bone destruction was reduced in the $100 \mu \mathrm{g}$ etanercept-treated mice compared to that in control CIA mice (Fig. 4A and B), suggesting that etanercept prevents not only destruction of focal bone adjacent to the joints, but also systemic bone destruction. Furthermore, during the early stage of the disease, these effects were observed only after etanercept treatment, indicating that etanercept treatment may be effective in preventing osteoporosis in patients with early RA.

Notably, the highest dose of $400 \mu \mathrm{g}$ etanercept did not reduce the symptoms of arthritis. The incidence of arthritis and the arthritis scores of mice treated with $400 \mu \mathrm{g}$ etanercept exceeded those of control CIA mice (Fig. 2A and B). Furthermore, pannus formation and cartilage damage in the joints of mice treated with $400 \mu \mathrm{g}$ etanercept were similar to those in CIA mice (Fig. 2C). The levels of sRANKL and TNF- $\alpha$ in sera of mice treated with $400 \mu \mathrm{g}$ etanercept did not decrease, and no anti-osteoporotic effect was detected in these mice (Fig. 2D and E).

Etanercept is considered effective for treating RA and has been traditionally prescribed to many RA patients. Numerous scientists and clinicians consider that an abnormal inhibition of etanercept activity by several known factors can explain the fact that some patients do not respond to this treatment. The development of ADA against etanercept could be one of these factors. However, etanercept was considered less likely to induce development of ADA in comparison to other monoclonal antibody drugs. Since the current literature does not unequivocally exclude the possibility of induction of an antietanercept antibody, in the present study we used an ELISA assay to detect this antibody (Fig. 2F and H). We clearly detected the anti-etanercept antibody only in the sera from the etanercept-refractory group $(400 \mu \mathrm{g})$. The recommended etanercept dose for humans is $714 \mu \mathrm{g} / \mathrm{kg}$; given that a mouse weighs $20 \mathrm{~g}$, this is equivalent to $\sim 178.5 \mu \mathrm{g} / \mathrm{mouse}$, considering the body surface area (21). Thus, $400 \mu \mathrm{g}$ of etanercept was an overdose for the mice, which might have led to the synthesis of the anti-etanercept antibody that may have caused the ineffectiveness of the high-dose etanercept treatment. Although the number of patients refractory to etanercept has continued to increase (22), the reason for this has not been elucidated. Several studies have suggested that the production of anti-TNF- $\alpha$ inhibitor antibodies is the main reason for treatment refractoriness (23-25). However, the frequency of the anti-etanercept antibody was reported at $<5.6 \%(26-27)$, considerably lower than that of other anti-TNF- $\alpha$ inhibitors (12-44\% anti-infliximab and 6-87\% anti-adalimumab) (28), and no correlation has been demonstrated between the presence of the anti-etanercept antibody and poor clinical response. In this study, we have shown that the production of anti-etanercept antibody by mice exposed to an etanercept overdose is associated with the inefficiency of the drug. We have shown that etanercept effectively reduces inflammation and focal and systemic osteoporosis, and that these effects partially result from the indirect inhibition of osteoclastogenesis. These effects were considerable, even when etanercept was only administered during the early stage of the disease, suggesting that etanercept treatment during early stages may be useful to ameliorate RA. We also demonstrated the presence of anti-etanercept antibody in the serum of etanercept treated-refractory mice, which provides a clue to the potential mechanism of resistance in patients with RA who are refractory to treatment.

\section{Acknowledgements}

We are very grateful to Karin for professional proofreading. This study was supported by a grant of the Korea Healthcare Technology R\&D project, Ministry for Health, Welfare \& Family Affairs, Republic of Korea (A092258).

\section{References}

1. Aletaha D, Neogi T, Silman AJ, et al: 2010 rheumatoid arthritis classification criteria: an American College of Rheumatology/European League Against Rheumatism collaborative initiative. Ann Rheum Dis 69: 1580-1588, 2010.

2. Taylor PC: Pharmacology of TNF blockade in rheumatoid arthritis and other chronic inflammatory diseases. Curr Opin Pharmacol 10: 308-315, 2010.

3. Scott DL and Kingsley GH: Tumor necrosis factor inhibitors for rheumatoid arthritis. N Engl J Med 355: 704-712, 2006.

4. Moreland LW, Schiff MH, Baumgartner SW, et al: Etanercept therapy in rheumatoid arthritis. A randomized, controlled trial. Ann Intern Med 130: 478-486, 1999.

5. Bathon JM, Martin RW, Fleischmann RM, et al: A comparison of etanercept and methotrexate in patients with early rheumatoid arthritis. N Engl J Med 343: 1586-1593, 2000.

6. Moreland LW, Baumgartner SW, Schiff MH, et al: Treatment of rheumatoid arthritis with a recombinant human tumor necrosis factor receptor (p75)-Fc fusion protein: N Engl J Med 337: 141-147, 1997.

7. Elliott MJ, Maini RN, Feldmann M, et al: Randomised double-blind comparison of chimeric monoclonal antibody to tumour necrosis factor alpha (cA2) versus placebo in rheumatoid arthritis. Lancet 344: 1105-1110, 1994.

8. van den Berg WB, Joosten LA and van de Loo FA: TNF alpha and IL-1 beta are separate targets in chronic arthritis. Clin Exp Rheumatol 17: S105-S114, 1999.

9. Kang KY, Lee KY, Kwok SK, et al: The change of bone mineral density according to treatment agents in patients with ankylosing spondylitis. Joint Bone Spine 78: 188-193, 2011.

10. Ju JH, Kang KY, Kim IJ, et al: Visualization and localization of rheumatoid knee synovitis with FDG-PET/CT images. Clin Rheumatol 27 (Suppl 2): S39-S41, 2008.

11. Wolfe F and Michaud K: Towards an epidemiology of rheumatoid arthritis outcome with respect to treatment: randomized controlled trials overestimate treatment response and effectiveness. Rheumatology (Oxford) 44 Suppl 4: iv18-iv22, 2005. 
12. National Institute for Health and Clinical Excellence: Adalimumab, etanercept and infliximab for the treatment of rheumatoid arthritis. http://www.nice.org.uk/nicemedia/live/11867/37914/37914.pdf. Accessed June 8, 2012.

13. Genovese MC, Bathon JM, Martin RW, et al: Etanercept versus methotrexate in patients with early rheumatoid arthritis: two-year radiographic and clinical outcomes. Arthritis Rheum 46: 1443-1450, 2002 .

14. O'Dell JR: Treating rheumatoid arthritis early: a window of opportunity? Arthritis Rheum 46: 283-285, 2002.

15. Ju JH, Cho ML, Jhun JY, et al: Oral administration of type-II collagen suppresses IL-17-associated RANKL expression of CD4 T cells in collagen-induced arthritis. Immunol Lett 117: 16-25, 2008

16. Yamaguchi N, Ohshima S, Umeshita-Sasai M, et al: Synergistic effect on the attenuation of collagen induced arthritis in tumor necrosis factor receptor I (TNFRI) and interleukin 6 double knockout mice. J Rheumatol 30: 22-27, 2003.

17. Kobayashi K, Takahashi N, Jimi E, et al: Tumor necrosis factor alpha stimulates osteoclast differentiation by a mechanism independent of the ODF/RANKL-RANK interaction. J Exp Med 191: 275-286, 2000.

18. Hsu H, Lacey DL, Dunstan CR, et al: Tumor necrosis factor receptor family member RANK mediates osteoclast differentiation and activation induced by osteoprotegerin ligand. Proc Natl Acad Sci USA 96: 3540-3545, 1999.

19. Boyle WJ, Simonet WS and Lacey DL: Osteoclast differentiation and activation. Nature 423: 337-342, 2003.

20. Lacey DL, Timms E, Tan HL, et al: Osteoprotegerin ligand is a cytokine that regulates osteoclast differentiation and activation. Cell 93: 165-176, 1998.
21. Reagan-Shaw S, Nihal M and Ahmad N: Dose translation from animal to human studies revisited. FASEB J 22: 659-661, 2008.

22. Finckh A, Simard JF, Gabay C and Guerne PA; SCQM physicians: Evidence for differential acquired drug resistance to anti-tumour necrosis factor agents in rheumatoid arthritis. Ann Rheum Dis 65: 746-752, 2006.

23. Haraoui B, Cameron L, Ouellet $M$ and White B: Antiinfliximab antibodies in patients with rheumatoid arthritis who require higher doses of infliximab to achieve or maintain a clinical response. J Rheumatol 33: 31-36, 2006.

24. Elkayam O, Burke M, Vardinon N, et al: Autoantibodies profile of rheumatoid arthritis patients during treatment with infliximab. Autoimmunity 38: 155-160, 2005.

25. Atzeni F, Doria A, Ghirardello A, et al: Organ-specific autoantibodies in patients with rheumatoid arthritis treated with adalimumab: a prospective long-term follow-up. Autoimmunity 41: 87-91, 2008.

26. Dore RK, Mathews S, Schechtman J, et al: The immunogenicity, safety, and efficacy of etanercept liquid administered once weekly in patients with rheumatoid arthritis. Clin Exp Rheumatol 25: 40-46, 2007.

27. Keystone EC, Schiff MH, Kremer JM, et al: Once-weekly administration of $50 \mathrm{mg}$ etanercept in patients with active rheumatoid arthritis: results of a multicenter, randomized, double-blind, placebo-controlled trial. Arthritis Rheum 50: 353-363, 2004

28. Emi Aikawa N, de Carvalho JF, Artur Almeida Silva C and Bonfá E: Immunogenicity of anti-TNF-alpha agents in autoimmune diseases. Clin Rev Allergy Immunol 38: 82-89, 2010. 